\title{
Ultrafine Copper Nanoparticles Exhibiting a Powerful Antifungal/Killing Activity Against Corticium Salmonicolor
}

\author{
Van Du Cao, Phuong phong Nguyen,,${ }^{\dagger, *}$ Vo Quoc Khuong, ${ }^{\dagger}$ Cuu Khoa Nguyen,,${ }^{\ddagger}$ Xuan Chuong Nguyen, \\ Cap Ha Dang, and Ngoc Quyen Tran ${ }^{\ddagger}$ \\ Department of Chemical and Food Engineering, Lac Hong University, Dong Nai Province, Vietnam \\ 'Department of Chemistry, University of Science, National University of Hochiminh City \\ *E-mail: ntpphong@hcmus.edu.vn \\ "Department of Materials and Pharmaceutical chemistry, Institute of Applied Materials Science, Vietnam Academy of Science and \\ Technology, HCMC 70000,Vietnam. *E-mail: tnquyen@iams.vast.vn \\ Received January 25, 2014, Accepted May 6, 2014
}

\begin{abstract}
In this paper ultrafine copper nanoparticles (CuNPs) were prepared from copper salt via chemical reduction method with sodium citrate dispersant and polyvinylalcol (PVA) capping polymer. The colloidal CuNPs were characterized by using UV-Visible spectroscopy, Transmission Electron Microscopy (TEM), and X-ray Diffraction (XRD) techniques. Our obtained results indicated that the CuNPs were produced ranging from 2 to $4 \mathrm{~nm}$ in diameter. The colloidal solution at $7 \mathrm{ppm}$ of CuNPs exhibited a powerful antifungal activity against Corticium salmonicolor (C. Salmonicolor). Fungal killing assays showed colloid solutions containing $10 \mathrm{ppm}$ of CuNPs killed entirely the cultured fungus. A highly killing activity against the fungus was also performed when the CuNPs were sprayed on pink disease-infected rubber trees. These positive results may offer a great potential to produce CuNPs-based eco-fungicide for pink disease.
\end{abstract}

Key Words : Ultrafine CuNPs, Sodium citrate, Polyvinylalcol, Corticium salmonicolor, Pink disease

\section{Introduction}

Use of metallic nanoparticles for biomedical and industrial applications has recently been much attention due to many novel properties such as optical, catalytic and antimicrobial as compared to their bulk metallic form. ${ }^{1-5}$ Among them, copper nanoparticles (CuNPs) exhibited interesting thermal and electrical conductivity, antifriction and antimicrobial property resulting in several practical applications such as heat exchangers, conductive copper lines in inkjet printing, antifriction additive in lubricant and antimicrobial agent. ${ }^{6-14}$ Several prepared methods of the CuNPs have been reported such as chemical method, thermal decomposition, microwave heating, microemulsion techniques, etc. ${ }^{15-19}$ It is well-known that at small sizes, CuNPs and other metallic nanoparticles offer special chemical, physical and biological properties due to an increase in surface area of the nanoparticles. Therefore preparation of small-sized CuNPs has been much attractive. .0-22 $^{2}$

Several kinds of metallic nanoparticles like silver, copper and gold exhibiting strong activity against some microbes and microorganisms. Among of them, nanosilver has been the most studies and applications. The nanoparticles also performed a strongly antifungal activity against various plant pathogenic fungi such as Phytophthora, C. Salmonicolor, etc. ${ }^{23}$ However, studies on fungal activity of CuNPs have recently received attention because the nanoparticles are much cheaper than that of Ag or AuNPs. This advantage could offer the antifungal applications of the CuNPs in agriculture. Up to now, antifungal activity of CuNPs has just been evaluated on a few of microbes but there has not been done with microorganisms like $C$. Salmonicolor ${ }^{12-14}$ The $C$. Salmonicolor microorganism causes pink disease on rubber and wood trees in tropical regions. This disease causes damage to the trunk and branches of the rubber trees resulting in a significant reduction of latex yield. ${ }^{24}$

In this study, ultrafine CuNPs were prepared by chemical reduction method in the presence of the sodium citrate dispersant and polyvinylalcol (PVA) capping polymer. UVVis spectroscopy, TEM and XRD technique were used to characterize CuNPs. Antifungal activity against $C$. Salmonicolor on petri dishes and rubber field were then evaluated.

\section{Experimental}

Materials. Copper(II) chloride dihydrate $\left(\mathrm{CuCl}_{2} \cdot 2 \mathrm{H}_{2} \mathrm{O}\right.$, 99.0\%), Glycerol $\left(\mathrm{C}_{3} \mathrm{H}_{5}(\mathrm{OH})_{3}, 99.0 \%\right)$, trisodium citrate dihydrate $(99.0 \%)$, hydrazin dihydrate $\left(\mathrm{N}_{2} \mathrm{H}_{4} \cdot 2 \mathrm{H}_{2} \mathrm{O}, 50 \%\right)$ were purchased from Acros Organics. Polyvinyl alcohol (PVA, $\mathrm{Mw}=60.000 \mathrm{~g} / \mathrm{mol}$ ) was obtained from Merck. All other reagents were used without further purification. C. salmonicolor was isolated from pink disease-infected rubber trees at Dong Nai Rubber Company (Dong Nai province-Vietnam).

Synthesis of CuNPs with Dispersant and Capping Polymer. Colloidal CuNPs (220 ppm of the nanoparticles) were prepared with a simple method in a short period of time that could scale up for practical production. Briefly, the capping polymer (PVA) $2 \mathrm{~g}, \mathrm{CuCl}_{2}(0.1 \mathrm{M}, 10.4 \mathrm{~mL}$; copper salt/PVA $=7 \mathrm{wt} / \mathrm{wt} \%)$ and trisodium citrate $(0.1 \mathrm{M}, 4.8 \mathrm{~mL})$ were dissolved in $275 \mathrm{~mL}$ glycerol. Mixture was heated to 
predeterminated temperature by using a microwave oven (Sanyo EM-S2088W, 800W). Once the mixture reached the set temperature (around $80 \mathrm{Sec}$ ), the oven was turn-off and hydrazine hydrate solution $(0.1 \mathrm{M}, 10.4 \mathrm{~mL})$ was immediately added dropwise to the mixture under stirring. The reaction mixture was stopped to obtain a colloidal CuNPs after one minute of the addition completed. The colloidal CUNPs was characterized by UV-Vis, TEM and XRD.

Characterization of CuNPs. The UV-Vis absorption spectrum of the colloidal CuNPs solution was measured by Jasco V670. TEM images were measured by JEM-1400. The samples were prepared by dropping CuNPs solution onto a carbon-coated copper grid. XRD result was characterized using D8 advanced Bragg X-ray with $\mathrm{Cu} \mathrm{K} \alpha$ radiation. For sample handling, glass slide was used as a substrate for measurement. Leaned substrate was covered with the colloidal CuNPs and dried in air.

Determination of Antifungal Activity. The antifungal activity against $C$. salmonicolor was evaluated by using the in vitro plate dilution method. The colloidal CuNPs were mixed with $25 \mathrm{~mL}$ of melting Potato Dextrose Agar (PDA) medium then were poured into Petri dishes with final concentration of 3, 5 and $7 \mathrm{ppm}$. The control dishes contained distilled water instead of the colloidal CuNPs. The fungus was transferred equally onto center point of the prepared petri dishes and incubated at room temperature for 6 days. The growth inhibition of the fungus was evaluated by measuring diameter of colony growth and calculated with formula: growth inhibition $(\%)=\left(\mathrm{d}_{1}-\mathrm{d}_{2} / \mathrm{d}_{1}\right) \times 100$, where $\mathrm{d}_{1}$ and $\mathrm{d}_{2}$ are diameters of the colony of control and CuNPscontained samples, respectively. All experiments were performed in triplicate.

Determinaton of Fungal Killing Ability. C. salmonicolor was grown on potato dextrose agar (PDA) medium at room temperature for 7 days. Samples of with CuNPs concentration of 7 and $10 \mathrm{ppm}$ were sprayed directly on each dish one or two times $(0.5 \mathrm{~mL}$ per dish). The fungal killing ability was assessed after $24 \mathrm{~h}$. If the fungus had not been collapsed, the fungal petri dishes were sprayed second time then reassessed after $24 \mathrm{~h}$.

\section{Results and Discussion}

Characterization of CuNPs. Preparation of the CuNPs in the presence of PVA capping polymer and citrate dispersant was done at different ratios of copper salt and PVA (5 and $7 \mathrm{wt} / \mathrm{wt} \%$ ). Color of the CuNPs colloidal solutions are slightly difference as shown in Figure 1 that may indicate a difference in size of produced CuNPs in two colloidal solutions. This could be predicted from UV-Vis spectroscopy of two colloidal solutions in which peaks appear at $562 \mathrm{~nm}$ and $558 \mathrm{~nm}$ for the prepared CuNPs samples stabilized with 5 and $7 \mathrm{wt} / \mathrm{wt} \%$ of PVA, respectively (Figure 2 ), In the figure, a broadened absorbance region from short wavelength to $558 \mathrm{~nm}$ or $562 \mathrm{~nm}$ are reported as the surface Plasmon of a ultrafine CuNPs colloidal solution. ${ }^{19,25}$

Figure 3(a) and 3(b) show TEM images of the prepared

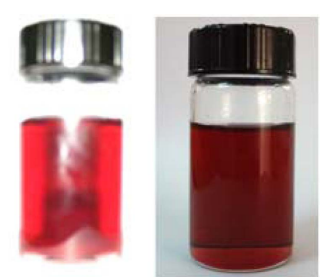

Figure 1. Color of colloidal solutions 5 (left) and $7 \mathrm{wt} / \mathrm{wt} \%$ (right) of CuNPs.

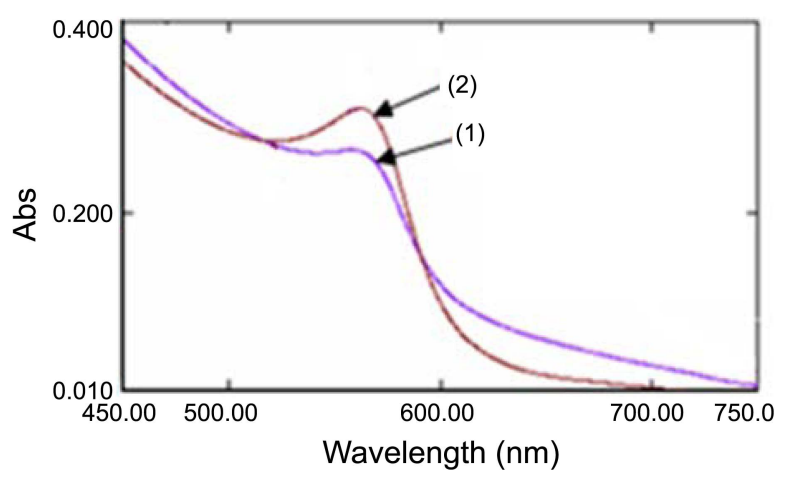

Figure 2. UV-Vis absorbance of the colloidal solutions at different ratio of copper salt and PVA: 5(1) and $7 \mathrm{wt} / \mathrm{wt} \%$ (2).

CuNPs in which CuNPs ranging 2-3 $\mathrm{nm}$ in diameter are prepared at $5 \mathrm{wt} / \mathrm{wt} \%$ of copper salt and PVA and histogram shows a narrow size distribution (Figure 3(a)). The result from TEM observation accorded with results from UV-Vis measurement of the colloidal solutions in which a broadened region of the surface Plasmon was observed for CuNPs at 2 $\mathrm{nm}$ in diameter. ${ }^{20}$ When ratio of copper salt and PVA was
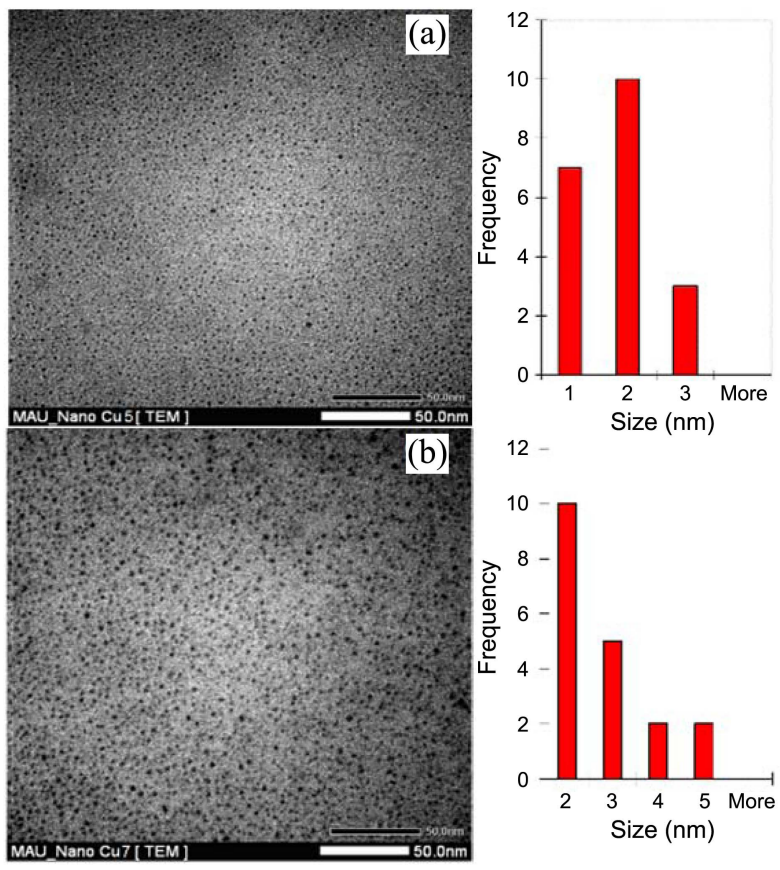

Figure 3. TEM images and their size distribution histogram of the produced CuNPs at different ratio of copper salt and PVA: 5 (a) and $7 \mathrm{wt} / \mathrm{wt} \%$ (b). 
used at $7 \mathrm{wt} / \mathrm{wt} \%$, the produced CuNPS size are ranging from 2 to $5 \mathrm{~nm}$ (Figure 3(b)). Surface Plasmon resonance of the colloidal CuNPs was also observed but absorbance of surface Plasmon resonance was partially decreased due to slightly increase nanosize of the CuNPs. An additional experiment was conducted with ratio of copper salt and PVA at $10 \mathrm{wt} / \mathrm{wt} \%$, the obtained CuNPs was around $15 \mathrm{~nm}$ in diameter (data not shown here). These experiments indicated that increase in ratio of copper salt and PVA contributed to the increase in diameter of obtained CuNPs. That could be explained that the increment CuNPs produced resulting in reduction of PVA-protecting efficiency and increased CuNPs aggregation. According to a previous report, CuNPs could obtain in a smaller size under protection of PVA as compared to polyvinylpyrolydone (PVP)-protecting polymer at the same condition. The result could be explained that PVA and PVP possess hydroxyl groups or amide groups that can form complex with transition metals. However, higher concentration of hydroxyl groups presents in PVA when compared to amide groups in PVP. ${ }^{26}$ Moreover, aqueous solution of PVA is more viscous than that of PVP at the same polymer concentration. These result in reducing CuNPs size growth or aggregation in PVA.

Purity and stability of the CuNPs produced at the condition which were tested by XRD. The Figure 4 doesn't show any peak of copper oxides but appears only metallic copper with high crystallinity at 43.2, 50.4 and $74,0^{\circ}$ in XRD pattern. These peaks correspond to the typical face centered cubic of copper with miller indices at (111), (200) and (220), respectively. ${ }^{27}$ In some previous reports, depending on synthetic methods or solvents, copper oxides $\left(\mathrm{Cu}_{2} \mathrm{O}\right.$ and $\left.\mathrm{CuO}\right)$ could form in preparation of CuNPs. In these cases, a strongest peak of each copper oxide appears around $36^{\circ}\left(\mathrm{Cu}_{2} \mathrm{O}\right)$ and $38^{\circ}(\mathrm{CuO})$ in XRD patterns. ${ }^{28,29}$ Our obtained results confirm that the prepared CuNPs are high purity. The colloidal solution didn't change color after one month without storage in any inner gas (Image not shown here).

Antifungal Activity Against $\boldsymbol{C}$. salmonicolor. Colloidal solutions (particles at $2-5 \mathrm{~nm}$ in diameter) containing 220 ppm of CuNPs were diluted into colloidal solutions: 3 ppm, $5 \mathrm{ppm}$ and $7 \mathrm{ppm}$ for evaluating antifungal activity. Figure 5

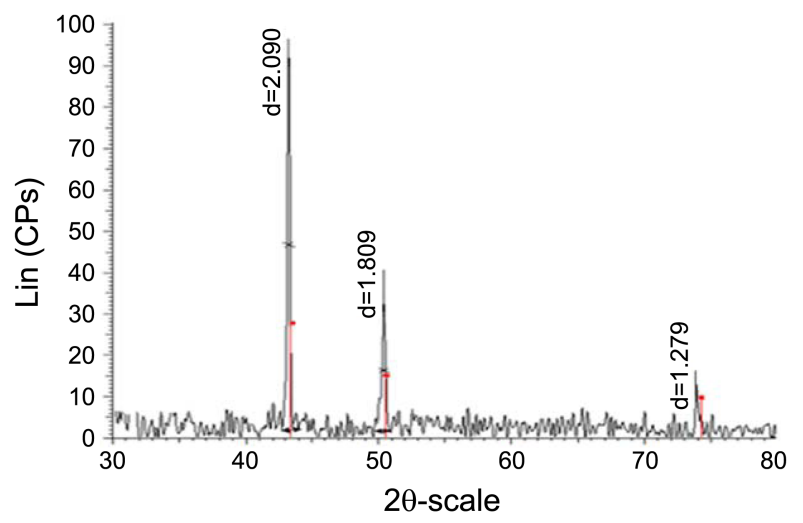

Figure 4. XRD pattern of the obtained CuNPs at the synthetic condition ( $5 \mathrm{wt} / \mathrm{wt} \%$ of copper salt and PVA).

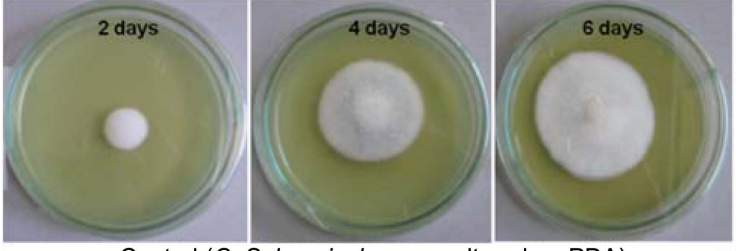

Control (C. Salmonicolor was cultured on PDA)

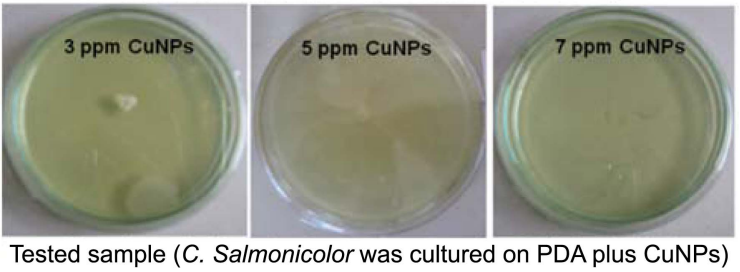

Figure 5. C. salmonicolor on media after 6 days culture (top panel); C. salmonicolor on media contained CuNPs after 6 days culture (bottom panels).

shows powerful antifungal ability against $C$. salmonicolor at CuNPs concentration of $7 \mathrm{ppm}$ at which $100 \%$ of the fungus was inhibited to grow according to formula for growth inhibition. At the lower concentrations of $3 \mathrm{ppm}$ and $5 \mathrm{ppm}$, CuNPs were less effective with $90.54 \%$ and $95.60 \%$ of fungal growth inhibition, respectively. Strong antimicrobial activity of CuNPs has recently been reported that released copper ion can cause microbial membrane damage. ${ }^{30}$

The result is very interesting for antifungal application of the ultra CuNPs. Bui et al. investigated antifungal activity of silver nanoparticles ( $7 \mathrm{~nm}$ in diameter) against $C$. salmonicolor, they reported that $50 \%$ of the fungus was inhibited growth at $27.2 \mathrm{ppm}$ of the nanoparticles. However, at the same study, 98.98 and $99.99 \%$ of E. coli growth inhibition obtained when the fungus was cultured on PDA media containing 5 and $7 \mathrm{ppm}$ of the silver nanoparticles, respectively. ${ }^{30}$ Different behavior of these $\mathrm{Ag}$ and $\mathrm{Cu}$ nanoparticles in antifungal activity against $C$. salmonicolor may be due to ultrafine nanosize of CuNPs providing a powerful antifungal activity or differences in organisation, structure and function of microbes. ${ }^{31,32}$

Fungal Killing Ability. According to results from antifungal activity studies, the colloidal solutions containing 7 ppm of CuNPs was selected for spraying on $C$. salmonicolor that had been cultured for 7 days. Figure 6 shows that most of the fungus was killed after spraying second time at the CuNPs concentration of $7 \mathrm{ppm}$. When CuNPs concentration increased up to $10 \mathrm{ppm}$ the fungal killing ability was increased. Figure 7 shows that all fungus was killed after 1 st spraying.

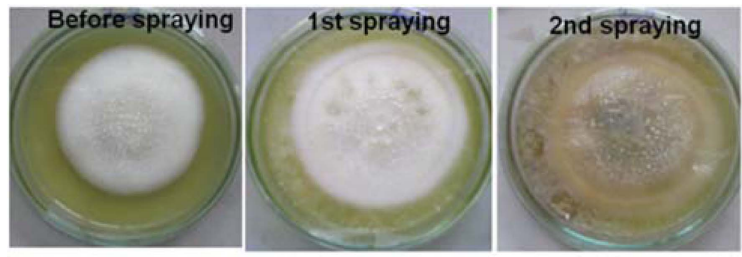

Figure 6. C. salmonicolor was killed by 7 ppm of CuNPs. 


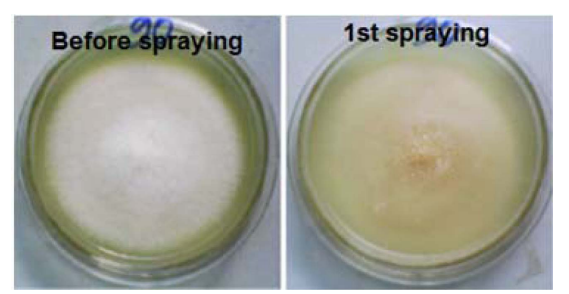

Figure 7. C. salmonicolor was killed by spraying $10 \mathrm{ppm}$ of a colloidal CuNPs solution.

These results indicated that fungal killing ability of CuNPs was more effective at a higher concentration of (10 ppm) in comparison with antifungal test (7 ppm). It could be explained due to the outgrowth of $C$. salmonicolor in fungal killing test, it was necessary to spray a higher CuNPs concentration to kill the C. salmonicolor.

Evaluation of the antimicrobial activity and fungal killing ability is very significant in our study because we have strongly expected to produce an ecofungicide for treating pink disease on rubber trees. It is well-known that the $C$. Salmonicolor causes pink disease on rubber fields in Vietnam. It causes damage to the trunk and branches resulting in a significant reduction of latex yield.

In addition, a preliminary result obtained from treating diseased rubber trees with ultrafine CuNPs showed that disease index reduced significantly after 2 times spraying. The obtained efficacy was similar with Validacin $5 \mathrm{~L}$ treatment ( $2 \mathrm{wt} \%$, current fungicide for pink disease on rubber trees). The experiment has been ongoing to evaluate fungal killing ability of CuNPs and protecting ability for rubber fields from C. salmonicolor. Our project has been expecting to pave the way for use of ultrafine CuNPs as an ecofungicide for rubber fields.

\section{Conclusion}

In this work, the ultrafine colloidal CuNPs (ranging from 2 to $5 \mathrm{~nm}$ in diameter) were prepared via chemical reduction method in the presence of PVA capping polymer and citrate dispersant. The method could produce the ultrafine CuNPs at a high concentration and the high stability. The nanoparticles exhibited a powerful antifungal activity against Corticium salmonicolor and high killing ability at concentration of $7 \mathrm{ppm}$ and $10 \mathrm{ppm}$, respectively. Positive results from spraying the ultrafine CuNPs on pink disease-infected rubber trees promise its potential application as a cheap ecofungicide for agriculture.

Acknowledgments. This research is funded by Department of Science and Technology-Dongnai province No: 09/ HĐ-SKHCN.

\section{References}

1. Sharon, M.; Choudhary, A. K.; Kumar, R. J. Phytolog. 2012, 4, 83.

2. De, M.; Ghosh, P. S.; Rotello, V. M. Adv. Mat. 2008, 22, 4225.

3. Duan, Z.; Ma, G.; Zhang, W. Bull. Korean Chem. Soc. 2012, 33, 4003.

4. Huang, H. H.; Yan, F. Q.; Kek, Y. M.; Chew, C. H.; Xu, G. Q.; Ji, W.; Oh, P. S.; Tang, S. H. Langmuir 1997, 13, 172.

5. Abhilash, M. Int. J. Pharm. Bio. Sci. 2010, $1,1$.

6. Liu, M. S.; Lin, M. C. C.; Tsai, C. Y.; Wang, C. C. Int. J. Heat Mass Tran. 2006, 49, 3028.

7. Nada, E. A.; Masoud, Z.; Hijazi, A. Int. Commun. Heat Mass 2008, 35, 657

8. Yu, W.; France, D. M.; Routbort, J. L.; Choi, S. U. S. Heat Tran. Eng. 2008, 29, 432.

9. Li, D.; Sutton, D.; Burgess, A.; Graham, D.; Calvert, P. J. Mater. Chem. 2009, 19, 3719.

10. Tarasov, S.; Kolubaev, A.; Belyaev, S.; Lerner, M.; Tepper, F. Wear 2002, 252, 63.

11. Ruparelia, J. P.; Chatterjee, A. K.; Duttagupta, S. P.; Mukherji, S. Acta Biomater. 2008, 4, 707.

12. Drelich, J.; Li, B.; Bowen, P.; Hwang, J. Y.; Mills, O.; Hoffman, D. Appl. Surf. Sci. 2011, 257, 9435.

13. Rastogi, L.; Arunachalam, J. Colloid Surf. B 2013, 108, 134.

14. Raspolli Galletti, A. M.; Antonetti, C.; Marracci, M.; Piccinelli, F.; Tellini, B. Appl. Surf. Sci. 2013, 280, 610.

15. Biçer, M.; Şişman, İ. Powder Technol. 2010, 198, 279

16. Nasibulin, A. G.; Ahonen, P. P.; Richard, O.; Kauppinen, E. I.; Altman, I. S. Nanopart. Res. 2001, 3, 383.

17. Zhu, H.; Zhang, C.; Yin, Y. Nanotechnology 2005, 16, 3079.

18. Blosi, M.; Albonetti, S.; Dondi, M.; Martelli, C.; Baldi, G. J. Nanopart. Res. 2011, 13, 127.

19. Pham, L. Q.; Sohn, J. H.; Park, J. H.; Kang, H. S.; Lee, B. C. J. Colloid Interf. Sci. 2012, 365, 103.

20. Zhang, H. X.; Siegert, U.; Liu, R.; Cai, W. B. Nanoscale Res. Lett. 2009, 4, 705.

21. Mott, D.; Galkowski, J.; Wang, L.; Luo, J.; Zhong, C. Langmuir 2007, 23, 5740.

22. Kwon, J. W.; Yoon, S. H.; Lee, S. S.; Seo, K. W.; Shim, I. W. Bull. Korean Chem. Soc. 2005, 26, 837.

23. Dang, V. P.; Vo, T. K. L.; Nguyen, T. K. L.; Nguyen, N. D.; Nguyen, D. C.; Bui, D. D.; Bui, D. C.; Nguyen, Q. H. J. Exp. Nanosci. 2010, 5, 169.

24. Xiong, J.; Wang, Y.; Xue, Q.; Wu, X. Green Chem. 2011, 13, 900.

25. Cao, V. D.; Tran, N. Q. Nguyen, T. P. P. J. Exp. Nanosci. DOI:10.1080/17458080.2013.848298.

26. Garces, L. J.; Hincapie, B.; Makwana, V. D.; Laubernds, K.; Sacco, A.; Suib, S. L. Micropor. Mesopor. Mat. 2003, 63, 11.

27. Slavati-Niasari, M.; Davar, F.; Mir, N. Polyhedron 2008, 27, 3514.

28. Yu, E.; Piao, L.; Kim, S. H. Bull. Korean Chem. Soc. 2011, 32, 4099.

29. Song, X.; Sun, S.; Zhang, W.; Yin, Z. J. Colloid Interf. Sci. 2004, 273, 463.

30. Bagchi, B.; Kar, S.; Dey, S. K.; Bhandary, S.; Roy, D.; Mukhopadhyay, T. K.; Das, S.; Nandy, P. Colloid Surf. B 2013, 108, 358.

31. Shrivastava, S.; Bera, T.; Roy, A.; Singh, G.; Ramachandrarao, P.; Dash, D. Nanotechnology 2007, 18, 103.

32. Tiwari, D. K.; Behari, J.; Sen, P. Curr. Sci. 2008, 95, 647. 